\title{
XXXVIII. Improvements in the hüfner type of spectrophotometer
}

\section{F. Twyman}

To cite this article: F. Twyman (1907) XXXVIII. Improvements in the hüfner type of spectrophotometer , Philosophical Magazine Series 6, 13:76, 481-488, DOI:

10.1080/14786440709463623

To link to this article: http://dx.doi.org/10.1080/14786440709463623

曲 Published online: 16 Apr 2009.

Submit your article to this journal $₫$

Џ Article views: 2

Q View related articles $₫$ 


\section{[ 481 ]}

XXXVIII. Improvements in the Hiifner Type of Spectrophotometer. By F. Twчmax *.

T $\mathrm{HE}$ following is a description of a Spectrophotometer of 1 the Hüfner type to which an addition has been made which converts it into a Polarimeter, by means of which optical rotations can be measured for light of any wavelength in the visible part of the spectrum. Incidentally an improvement in design is described whereby greater accuracy is attained.

Fig. 1.

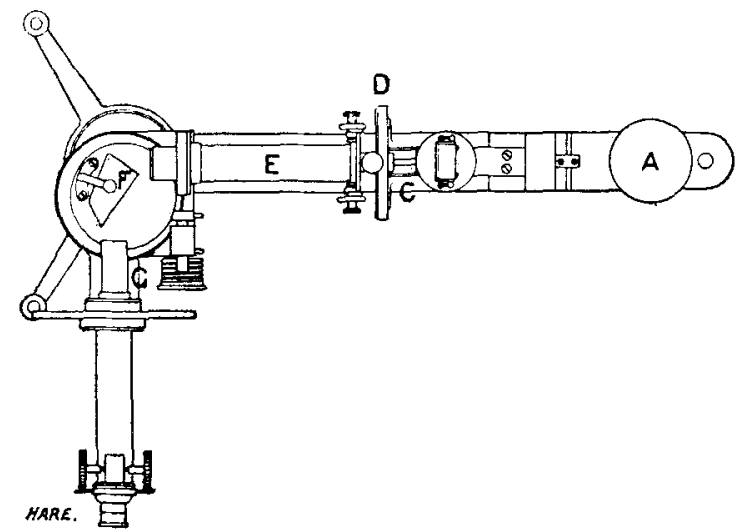

Fig. 2.

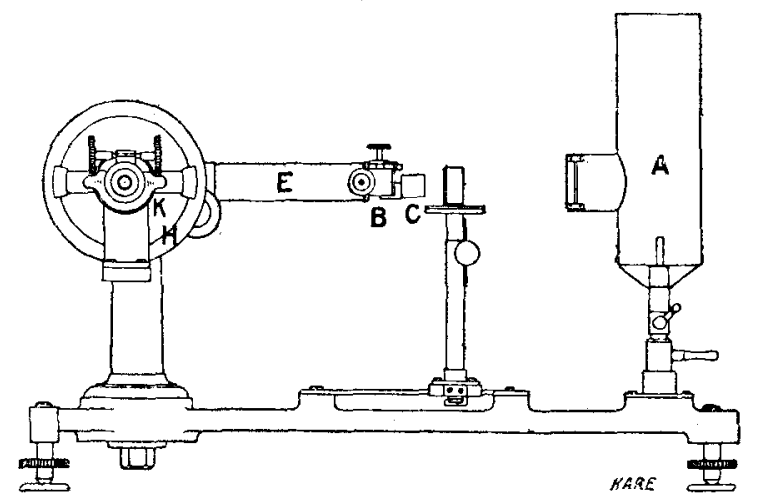

The Spectrophotometer part of the apparatus (shown in plan and elevation in figs. 1 and 2 and in fig 3) was designed and constructed in the workshop of Messrs. Adam Hilger Ltd.,

* Communicated by the Physical Society : read January 25, 1907. Phil. Mag. S. 6. Vol. 13. No. 76. April 1907. 2 L 
for Dr. C. E. Kenneth Mees in 1904, and embodies many suggestions of his and of Mr. S. E. Sheppard's. The Optical Train is the same as that of the Hüfner Spectrophotometer

Fig. 3.

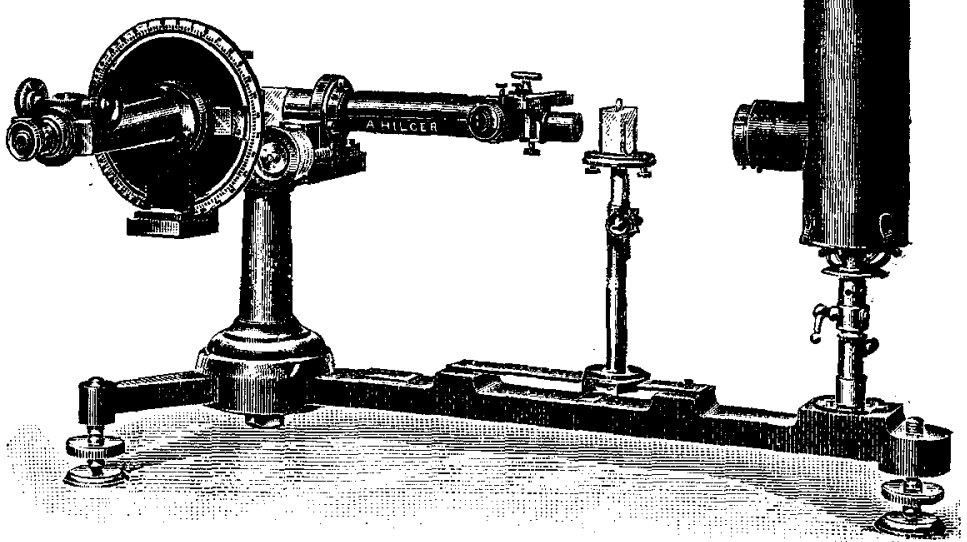

as made by Albrecht; with the exception that instead of the dispersion prism being the usual prism of $60^{\circ}$, the now fairly well-known constant-deviation prism is employed.

Fig. 4.

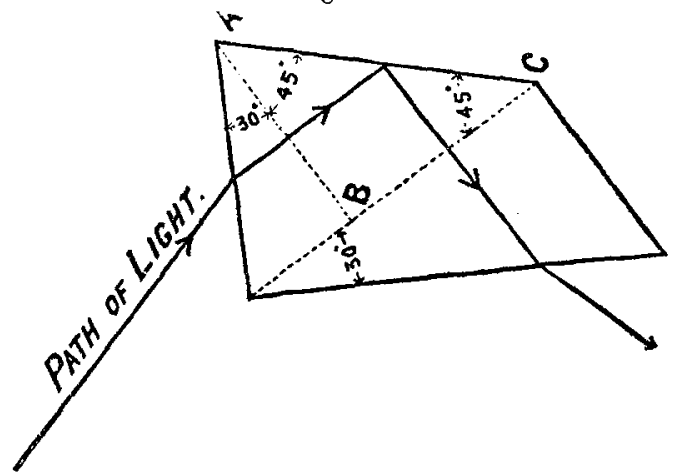

The prism is shown in fig. 4. It may be considered as built up of two $30^{\circ}$ prisms and one right-angled prism from the hypotenuse of which light is internally reflected as shown in the figure. The prism is, however, made in one piece. This construction of prism has the very important mechanical advantage that the telescope and collimator are both fixed, being rigidly attached to a strong cast-iron base. The passing 
from one part of the spectrum to another is effected by rotation of the prism by a micrometer screw.

The light source, A, is fixed to the cast-iron bed of the instrument. The light passes partly above and partly through a polarizing prism $\mathrm{C}$. The upper beam passes first through

Fig. 5.

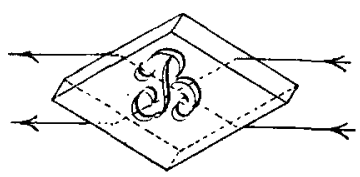
the substance whose absorption is to be measured and then through a thin wedge of neutral-tint glass, by the lateral trauslation of which the two beams may be made equal in intensity. Both beams then pass through the rhomb of glass B (fig. 5) by means of which the two beams are brought into elose juxtaposition. Immediately behind this rhomb is the slit of the spectroscope, the edge of the rhomb and the jaws of the slit both being in focus simultan fously when one observes them in the telescope.

The light after collimation in the usual way then undergoes dispersion by the constant-deviation prism, and passes on to the second polarizing prism $G$, and so on to the telescope. The second polarizing prism is mounted on a circle $\mathrm{H}$ divided into degrees and reading by a vernier to minutes, and by rotation of this the polarized beam of light can be reduced in intensity in the usual way till the intensity of the top and bottom beams is equal; and the density of the absorbing substance under test is then determined by means of the $\cos ^{2}$ law (the intensity of the light passing through crossed nicols being proportional to the square of the cosine of the angle between their planes of polarization).

The instrument as used as a spectrophotometer will be found very completely described (together with an admirable bibliography on the subject of Spectrophotometry) in a paper by Messrs. C. E. Kenneth Mers and S. E. Sheppard printed in the 'Photographic Journal' for July 1904.

I might, however, again mention here the fact that the wave-length of the light under observation is read off direct on the helical drum of the micrometer-screw, see fig. 1 . This is an arrangement which is found of great convenience in practice. The wave-lengths ${ }_{o}$ can be relied on to an average accuracy of well within 10 Angström units.

The present paper is principally concerned

(A) with the mode of correction of a serious error to which polarization spectrophotometers are liable;

(B) with the use of the instrument as a polarimeter.

(A) The error referred to is that due to the polarization of the light on transinission through the dispersing prism, $2 \mathrm{~L} 2$ 
whether it be an ordinary prism of $60^{\circ}$ or other angle, or a constant-deviation prism as in the present instrument.

Considering the case where a beam of light, collimated but initially unpolarized, is passed through the prism. Such a beam is the upper beam in the present instrument, which passes over the first nicol. The beam when it emerges is partially polarized, owing to the unequal reflexion of vibrations in and at right angles to the plane of incidence.

(In the case of a beam initially polarized in a plane neither in nor at right angles to the plane of incidence, matters are further complicated by the reflexion within the prism, which causes elliptical polurization; a state of things which has to be avoided by very carefully setting up the first nicol, until it is found that satisfactory extinction of the polarized beam can be obtained by rotation of the second nicol.)

Considering the appearance of the field of view as seen in the eyepiece, in the zero position of the second nicol: the position, namely, in which its plane of polarization is paralle! to that of the first nicol. One sees a slit of light wider or narrower according to the width to which one may think it advisable to open the shutters in the eyepiece which limit the field of view. This slit of light is bisected by a fine horizontal line, the edge of the rhomb in front of the slit, which is almost invisible when the intensities above and below are equalized by the adjustment of the wedge of neutral-tint glass. The light-absorbing medium, whose density it is required to measure, is introduced into the top beam, and the second nicol is then rotated to obtain equality of intensity. The lower half varies in intensity according to the $\cos ^{2}$ law; but owing to the polarization due to the dispersion prism the top beam also varies in intensity, and the absorption if determined simply by the application of the $\cos ^{2}$ law will consequently be incorrect. The amount of the error can be determined by applying Fresnel's equations to any particular case. We will consider the actual state of things in the original instruments made, i. e., a prism of refractive index 1.658 for the light under observation.

Let $\theta$ and $\theta_{1}$ represent respectively the angles of incidenco and refraction at the surface of the dispersion prism.

According to Fresnel's equations, if unpolarized light of intensity $a^{2}$ fall on a reflecting surface at an angle of incidence $\theta$, and if $\theta_{1}$ represent the angle of refraction, the intensity of that part of the reflected light which is polarized 
in the plane of incidence is (see Schuster's 'Theory of Opties,' first edition, p. 49)

$$
\frac{1}{2} a^{2} \frac{\sin ^{2}\left(\theta_{1}-\theta\right)}{\sin ^{2}\left(\theta_{1}+\theta\right)}
$$

The intensity of the transmitted light if that light be polarized in the plane of incidence will then be

$$
\frac{1}{2} a^{2}\left\{1-\frac{\sin ^{2}\left(\theta_{1}-\theta\right)}{\sin ^{2}\left(\bar{\theta}_{1}+\theta\right)}\right\} \text {. }
$$

As in a prism at the angle of minimum deviation the angle of incidence on entering the prism is equal to that of refraction on leaving it, the intensity of the transmitted light if that light be polarized in the plane of incidence, is, after passing right through the prism,

$$
\frac{1}{2} a^{2}\left\{1-\frac{\sin ^{2}\left(\theta_{1}-\theta\right)}{\sin ^{2}\left(\theta_{1}+\theta\right)}\right\}^{2} \text {. }
$$

For the case under consideration this works out to $\frac{a^{2}}{2} \times \cdot 651$.

The polarization due to the prism in the plane at right angles to this will bo found, when evaluated, to be negligible.

When, then, a spectrum produced by such a prism is observed through a polarizing prism, and the latter is rotated, the beam varies in intensity from 1 to 651 (for the particular wave-length for which the $\mu$ of the prism $=1 \cdot 658$ ).

It will be seen how extremely serious is this polarizing effect of the dispersion prism. Want of leisure has prevented me from reading up fully the bibliography of the subject, but although this error has been previously mentioned and seems fairly well known, there certainly seems to have been insufficient attention bronght to bear ou the subject, as there are still polarization photometers made which suffer from the full amount of this error.

The Hüfner instrument even in its original form is to a very great extent protected from this failing by the presence of the so-called Hüfner rhomb in front of the slit. It will be seen at once that this produces a partial polarization of the upper beam of light in a plane perpendicular to that of the partial polarization produced by the dispersion prism. By applying Fresnel's equations as in the case of the dispersion prism above, we can find the amount of this partial polarization. The rhombs in the first instrument made were of Jena borosilicate crown of $\mu_{D}=1.517$, the angle of the front and back 
edges of the rhomb being $70^{\circ}$ (which makes the angle of incidence of the beam upon the face of the rhomb $55^{\circ}$ ). With these values we find that the intensity of the light transmitted by the rhomb, if that light be polarized after transmission in the plane of incidence, would vary from 1 . to 0.732 : the partial polarization due to the rhomb in a plane at right angles to this being negligible.

Thus if the beam after passage through both rhomb and dispersion prism be polarized, it will vary from 0.732 to 0.651 as the analyzing nicol is turned.

This variation in the intensity of the top beam produces errors of the following amounts:-

Density of the absorbing medium under test

(i.e. $\log _{10} \frac{\mathrm{I}_{1}}{\mathrm{I}_{0}}, \mathrm{I}_{1}$ being the

intensity of the incident beam and $I_{0}$ that of the transmitted beam).

$0 \cdot 054$

0.231

$0 \cdot 602$

$1 \cdot 521$
Percentage error in the density, to be added or subtracted according as the first nicol is set with its plane of polarization vertical or horizontal.

$$
\begin{array}{r}
11 \cdot 5 \\
9 \cdot 4 \\
6 \cdot 4 \\
3 \cdot 3
\end{array}
$$

The errors of reading are considerably less than the errors in the above table, so that it is very important to get rid of the latter. This is done in the more recent instruments by the simple and obvious plan of making the rhomb of the same glass as the prism, and of such angles that the angles of incidence of the beam on its surfaces equal those on the surfaces of the dispersion prism.

The following table will show the effect of this in several actual instances. A piece of neutral-tint glass was retained as a standard, and its density measured on various instruments, some with the old form of rhomb and some with the new. The plane of polarization of the front nicol was sometimes vertical, sometimes horizontal.

It was in the testing of Nos. 2 and 3 that we first berame fully aware of the magnitude of the error.

Having calculated the amount on the hypothesis that the error was caused in the manner I have described, the front nicol was rotated through $90^{\circ}$, and an altered reading of the expected amount was obtained. On replacing the Hüner rhomb by a fresh one of the same glass as the dispersion-prism and of corresponding angles, a reading was obtained exactly the mean between the two former ones. (See No. 3 below.)

Several instruments were made before readings on a test density were taken, and instrument No. 4 was one of these 
sent back for repair. The opportunity was taken of replacing the rhomb, after which it gave the correct reading, although I have no actual record of the final test reading.

\begin{tabular}{|c|c|c|c|}
\hline $\begin{array}{l}\text { Spectrophoto- } \\
\text { meter number. }\end{array}$ & $\begin{array}{l}\text { Density of } \\
\text { standard glass } \\
\text { at W.L. 5550 } \\
\text { as given by the } \\
\text { spectrophoto- } \\
\text { meter. }\end{array}$ & $\begin{array}{l}\text { Error due to } \\
\text { polarization by } \\
\text { the Hüner } \\
\text { rhomb and dis- } \\
\text { persion prisms. } \\
\text { (calculated). }\end{array}$ & $\begin{array}{c}\text { Corrected } \\
\text { reading. }\end{array}$ \\
\hline $1 \ldots \ldots \ldots$ & $1 \cdot 81$ & +05 & 1.86 \\
\hline $2 \ldots \ldots \ldots$ & 1.92 & -.05 & 1.87 \\
\hline $\begin{array}{l}3 \\
3 \\
3\end{array} \ldots \ldots$ & 1.92 & -.05 & 1.87 \\
\hline $\begin{array}{c}\text { (nicol rotated } \\
\text { through } 90^{\circ} \text { ) }\end{array}$ & 1.84 & $+\cdot 05$ & 1.89 \\
\hline $\begin{array}{c}3 \\
\text { (new rhomb) }\end{array}$ & $1 \cdot 88$ & 0 & $1 \cdot 88$ \\
\hline $4 \ldots \ldots \ldots$ & 1.83 & +.05 & 1.88 \\
\hline $5 \ldots \ldots \ldots$ & 1.86 & 0 & 1.86 \\
\hline $6 \ldots \ldots \ldots$ & 1.86 & 0 & 1.86 \\
\hline
\end{tabular}

One of the great sources of error in polarization photometers is the difficulty of obtaining pure plane-polarized light, and to keep it plane-polarized so that when it arrives at the second nicol it can be entirely extinguished. The extinction of the last two instruments was so good, that by rotation of the second nicol from the zero position to the $90^{\circ}$ position, the intensity varied from about 1 to 1/10,000. Imperfect annealing of the optical train and strain by clamping or cementing, or flaws in the first nicol are the chief difficulties met with-they give considerable but not insurmountable difficulties, and are readily detected in the instrument. They all tend to make densities appear too high.

(B) The use of the instrument as a polarimeter was first suggested by me, I believe, to Dr. K. C. Browning in conversation. Some while after that he commissioned Messrs. Hilger to undertake the construction of one of these instruments with the suggested polarimeter addition, which consists merely in the extension of the telescope support sufficiently far to enable one to interpose between the dispersion prism and the second nicol the medium of which one desires to measure the optical rotation.

The readings are taken by putting into the top beam a 
suitable density. This density is matched by rotation of the second nicol before and after the interposition of the substance under test. The difference of readings gives, of course, the optical rotation for the part of the spectrum under test.

In the instrument exhibited room is provided for the interposition of a $100 \mathrm{~mm}$. tube for liquids, but there is of course no difficulty in extending this to any desired amount. It will be seen that one measures by reducing one half of the field to the intensity of the other half which is already fairly dark. Thus one would expect the sensitiveness to be somewhat less than that obtained by the use of polarizers, in which one half of the field decreases while the other half increases in intensity. But it is an exceedingly useful addition to the spectrophotometer and produces a polarimeter free from some serious defects of most instruments excepting those with the Lippisch and similar polarizers. Moreover it is, of course, suitable either for use by monochromatic light or for the measurement of optical rotations for different wave-lengths.

I may add that a photographic negative is the best neutraltint medium with which I am acquainted, and is what is employed when using the instrument as a spectro-polarimeter.

\section{The Density of the Ether. By Sir OLivfr LODGE *. Part I.}

1. THE ætherial constants, $\mu$ and $\kappa$,- the magnetic permeability and the electric inductivity of free space, have hitherto been of completely unknown value, both as to kind of quality and as to numerical magnitude; though the reciprocal of their geometric mean is well known to be a velocity, the velocity with which the rther transmits transverse waves of all lengths and intensities.

2. For many reasons I have been accustomed to think of $4 \pi \mu$ (the ungeometrical part of a coefficient of self-inductance) as of something which by legitimate analogy could be spoken of as æetherial density, and of $4 \pi / \kappa$ as of something which by similar material analogy must be expressible as ætherial elasticity or rigidity: this kind of rigidity being probably due to the æther's own intrinsic energy of constitution, and being explicable on a recondite hydrodynamical basis, as treated in various forms by Lord Kelvin.

3. I have also adduced reasons (in 'Modern Views of Electricity') for regarding the ther as exceedingly, perhaps infinitely, incompressible; this view being based in the first

* Communicated by the Author. 